\title{
TITLE:
}

\section{Adaptive quantum state estimation for dynamic quantum states}

\section{$\operatorname{AUTHOR}(\mathrm{S})$ :}

Nohara, Saki; Okamoto, Ryo; Fujiwara, Akio; Takeuchi, Shigeki

\section{CITATION:}

Nohara, Saki ... [et al]. Adaptive quantum state estimation for dynamic quantum states. Physical Review A 2020, 102(3): 030401.

ISSUE DATE:

2020-09

URL:

http://hdl.handle.net/2433/255110

\section{RIGHT:}

C2020 American Physical Society; 許諾条件に基づいて掲載しています $\circ$ 
PHYSICAL REVIEW A 102, 030401(R) (2020)

Rapid Communications

\title{
Adaptive quantum state estimation for dynamic quantum states
}

\author{
Saki Nohara $\odot,{ }^{1}$ Ryo Okamoto $\odot,{ }^{1}$ Akio Fujiwara $\odot,{ }^{2}$ and Shigeki Takeuchi $\odot{ }^{1, *}$ \\ ${ }^{1}$ Department of Electronic Science and Engineering, Kyoto University, Kyotodaigakukatsura, Nishikyo-ku, Kyoto 615-8510 Japan \\ ${ }^{2}$ Department of Mathematics, Osaka University, Machikaneyama, Toyonaka 560-0043 Japan
}

(Received 29 April 2020; accepted 21 July 2020; published 9 September 2020)

\begin{abstract}
We propose an adaptive quantum state estimation method for nonstationary quantum states and verify the method by numerical simulations and experimental investigations. Adaptive quantum state estimation provides an asymptotically optimal scheme for estimating an unknown input quantum state by updating the measurement configuration upon the detection of each quantum. However, previous methods are only valid for quanta with the same quantum state (a stationary state). By adopting the likelihood function for a fixed number of recent detection results, our sequential adaptive quantum state estimation allows quantum states changing in time to be estimated. The numerical simulation results and experimental demonstration using photons agree well with the theoretical predictions. This method will find applications in various fields where dynamically changing quantum states need to be estimated.
\end{abstract}

DOI: 10.1103/PhysRevA.102.030401

\section{INTRODUCTION}

Estimating quantum states is a fundamental issue in various fields such as quantum communication [1-5], quantum computation [6-8], and quantum sensing [9]. Because of the statistical nature of quantum mechanics, the accuracy of estimations of an unknown quantum state is limited by the number of samples [10]. Therefore, finding an optimal measurement to achieve the best accuracy is very important. In general, the optimal measurement depends on the unknown quantum state itself [10]. To solve this problem, Nagaoka proposed an adaptive quantum state estimation (AQSE) procedure [11-13]. In AQSE, for each measurement, the value of the parameter is estimated and the measurement system is then optimized according to the estimated value. Fujiwara mathematically proved that AQSE exhibits strong consistency and asymptotic efficiency $[14,15]$.

Recently, we have demonstrated AQSE for the linear polarizations of photons (one parameter) [16] and for a single polarization qubit (three parameters) [17]. Furthermore, we performed a high-speed AQSE experiment using a liquid crystal retarder [18]. Related experiments have investigated state estimation protocols using a two-step adaptive measurement [19-23] in which a preliminary measurement of part of the whole ensemble was used to determine the measurement configuration for the remainder of the ensemble. However, to the best of our knowledge, all the previously reported adaptive schemes [16-23] are only valid for photons with the same quantum state (a stationary state).

In this Rapid Communication, we report our proposal for sequential adaptive quantum state estimation (SAQSE). In the previous AQSE method, the likelihood function accumulates all the past information on the photon detection so that the

\footnotetext{
*takeuchi@kuee.kyoto-u.ac.jp
}

estimated parameter deviates from the latest quantum state of the input photons. In SAQSE, the likelihood function only contains information on a fixed number of the most recent detection results so that the estimated results explicitly follow the change in the input quantum states. An efficient implementation of the SAQSE protocol was tested by a numerical simulation and real experiments using photons. In the numerical simulation, we show that the estimated angles using SAQSE follow the true values with linear change, sinusoidal change, and sudden change. In the experiments, it was found that the estimated value agrees well with the true values changing in time (the method is consistent) and that the variances approximately achieve the Cramer-Rao bound for the given number of photons (the method is efficient).

\section{SAQSE}

Figure 1(a) shows the flowchart of AQSE, where $l_{n}(\theta)$ is the log-likelihood function, $n$ is the number of input photons, $\hat{\theta}_{n}$ is the estimated value, and $N$ is the total photon number. For details on the AQSE algorithm, see Ref. [16]. In what follows, we restrict ourselves to a one-dimensional quantum statistical model $\left\{\rho_{\theta} ; \theta \in \Theta(\subset \mathbb{R})\right\}$ of a smooth parametric family of density operators on a Hilbert space $\mathcal{H}$ having a one-dimensional parameter $\theta$, as in [16]. Our aim is to estimate the true value of the one-dimensional parameter $\theta$ using a quantum estimation scheme. We start with an arbitrary initial estimate $\hat{\theta}_{1}$, and set $l_{1}(\theta)=0$ (Preparation). In Process-1, the measurement apparatus (half-wave plates in the real experimental setup) is changed so that the measurement basis is ideal for the quantum state with parameter $\theta=\hat{\theta}_{1}$. Then, in Process-2 to 3 , the next photon is input to the measurement system and either of one of the detectors $(d=0$ or 1) is lit. Depending on which detector has been lit after we apply the measurement $M\left(\hat{\theta}_{n}\right)_{d}$, the log-likelihood function 
(a) Preparation

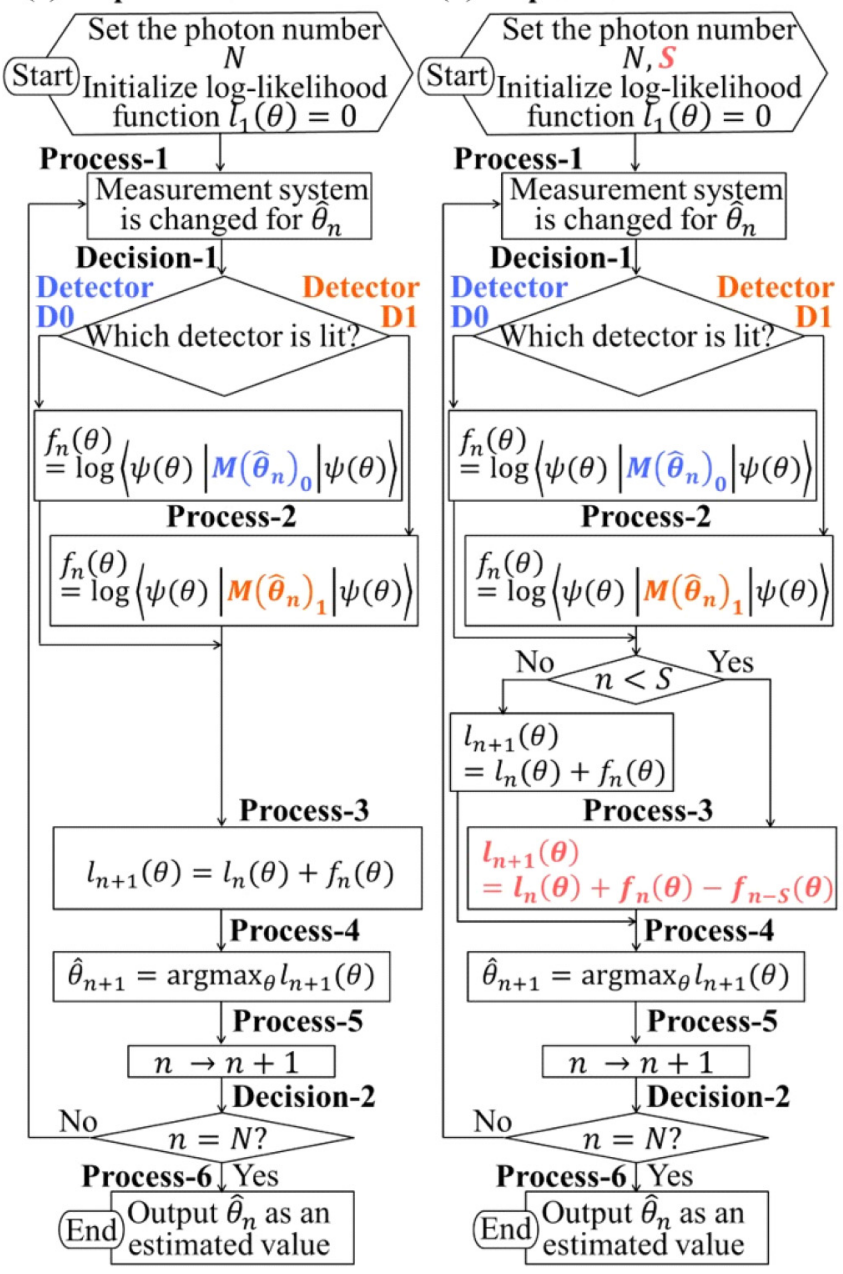

FIG. 1. (a) Flowchart of AQSE. (b) Flowchart of SAQSE.

$l_{n}(\theta)$ is updated using the following formula:

$$
l_{n+1}(\theta)=l_{n}(\theta)+f_{n}(\theta),
$$

where $f_{n}(\theta)=\log \left\langle\psi(\theta)\left|M\left(\hat{\theta}_{n}\right)_{d}\right| \psi(\theta)\right\rangle$. It should be noted that this update corresponds to adding information on the latest detection result in the log-likelihood function. Then, the new estimate $\hat{\theta}_{n+1}$ is calculated following the maximum likelihood method using $l_{n+1}(\theta)$ (Process-4). The above-mentioned process is repeated for the next input photon until the total number of photons reaches the preset value $N$.

We now more closely examine the updates of the loglikelihood function [Eq. (1)]. In AQSE, as explained above, the information of the $n$th measurement result $f_{n}(\theta)$ is "added" to the previous log-likelihood function $l_{n}(\theta)$. Expanding the recursive expression gives

$$
\begin{aligned}
l_{n+1}(\theta) & =l_{n}(\theta)+f_{n}(\theta) \\
& =f_{1}(\theta)+f_{2}(\theta)+\cdots+f_{n}(\theta) \\
& =\sum_{j=1}^{n} f_{j}(\theta) .
\end{aligned}
$$

It is clear that all the past information of the measurement results is retained by the latest $\log$-likelihood function $l_{n+1}(\theta)$.

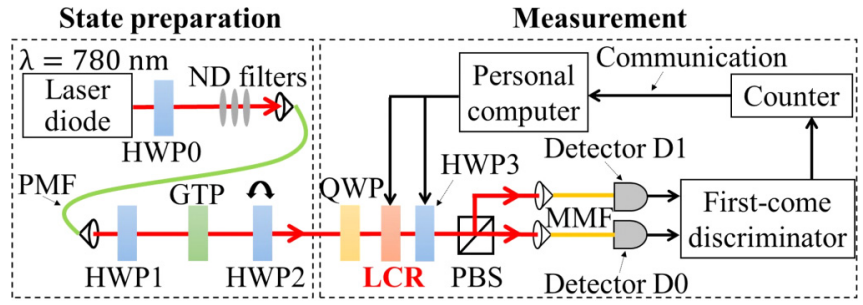

FIG. 2. Experimental setup for SAQSE.

Thus, it can be expected that for quantum states changing in time, the estimation result deviates from the latest "true value" due to the past measurement results persisting in the likelihood function.

A natural modification to solve this problem is to construct the likelihood function using the information of the latest $S$ measurement results, as follows:

$$
\begin{aligned}
l_{n+1}(\theta) & =f_{n-S+1}(\theta)+\cdots+f_{n}(\theta) \\
& =\sum_{j=n-S+1}^{n} f_{j}(\theta) .
\end{aligned}
$$

This modification can be efficiently implemented by replacing the equation used for Process-3 in Fig. 1(a) with the following equation:

$$
l_{n+1}(\theta)=\left\{\begin{array}{l}
l_{n}(\theta)+f_{n}(\theta), \quad n \leqslant S \\
l_{n}(\theta)+f_{n}(\theta)-f_{n-S}(\theta), \quad n>S,
\end{array}\right.
$$

using a tiny memory for the latest $S$ parameters $\left[f_{j}(\theta), j=\right.$ $n-S+1 \sim n]$. Note that parameter $S$, which determines how many recent measurement results need to be considered for the estimation, is set in terms of the rate of change of the input quantum state. In the actual measurement system, $S$ could also be adjusted according to the rate of change of the estimation results. Note also that in the following analysis we stick to Eq. (3), which corresponds to the simple moving average of $f_{n}(\theta)$. An alternative approach is to consider another equation corresponding to other averaging methods, e.g., the weighted moving average of $f_{n}(\theta)$.

\section{NUMERICAL SIMULATION OF SAQSE}

Following the experimental setup in Fig. 2, we numerically simulate and experimentally demonstrate SAQSE. In the simulation and experiment, we estimate the angle of the linear polarization of photons generated by a photon source that varies with time. In the state preparation part in Fig. 2, the light from a laser diode (wavelength $780 \mathrm{~nm}$ ) is strongly attenuated by a set of neutral density filters (ND filters), which are much smaller than the single photon level. Half-wave plate 0 (HWP0) is used to adjust the angle of the linear polarization to the axis of the polarization maintaining fiber (PMF). HWP1 is used to maximize the transmitted light of a Glan-Thompson prism (GTP). Then, the GTP with an extinction ratio of $10^{-5}$ is used to purify the polarization. HWP2 is used to change the angle of linear polarization of a single photon. In the measurement part in Fig. 2, the angle of HWP2 $(\theta)$ is estimated. The polarization of an input photon is analyzed by a quarter-wave plate (QWP), a liquid-crystal retarder (LCR), HWP3, and a 
polarizing beam splitter (PBS). We used the QWP to compensate the phase and HWP3 as a supplement to the LCR. For details, see Ref. [18]. After passing through the PBS, photons are guided to single-photon detectors D0 and D1 (SPCMAQR, Excelitas Technologies) on each PBS output port. Note that the detection efficiencies of the two detectors (D0 and D1) are balanced by inserting a small loss before the detectors. The outputs of the single-photon detectors are connected to a first-come discriminator, consisting of a custom-made electric circuit. When the discriminator receives the first signal from one of the detectors (D0 or D1) after the measurement for the $n$th photon starts, the discriminator reveals which detector has been clicked. The voltage of the LCR and the angle of HWP3 (measurement basis) for measuring the $(n+1)$ th photon are determined by calculating the maximum likelihood estimator $\hat{\theta}_{n+1}=\operatorname{argmax}_{\theta} l_{n+l}(\theta)$, the maximizer of the log-likelihood function, Eq. (3), chosen from among the 10000 points that divide the domain $[0,2 \pi)$ of the parameter $\theta$. When the change of measurement basis (LCR and HWP3) is completed, the measurement for the next $[(n+1)$ th $]$ photon will be started. Note that we randomly shift the measurement basis once every 30 times to suppress unexpected sudden changes of the estimated values, and discard the unexpected changes for the cases $\left|\hat{\theta}_{n}-\theta_{\text {true }}\right|>20^{\circ}$. The unexpected sudden change is due to the complete loss of the information obtained in the initial trials. It can be compensated by inserting measurements with a randomly determined measurement axis. When we inserted such a measurement once in 30 measurements, the probability of observing such a sudden change is less than $10^{-4}$.

In the following sections, we will show the results of the numerical simulation of SAQSE with various ways to change the true value.

\section{A. Simulation for linear changes of the true value}

First, we numerically simulate the case where the true value varies linearly with time. The amount of variation of the angle is $0.06^{\circ}$ for 300 input photons. The angle of the measurement basis (polarization) is changed according to the algorithm shown in Fig. 1(b). Figure 3(a) shows a single trajectory of the simulation result of SAQSE. The horizontal axis indicates the photon number $(n)$. The vertical axis shows the estimated angle of the linear polarization $\hat{\theta}_{n}$. The estimated values for SAQSE and AQSE are obtained along a single trajectory for 5000 input photons. This simulation result shows that the angle estimated by SAQSE [orange (gray) curve] follows the true value (black dashed line), changing slowly from $45.0^{\circ}$ to $46.0^{\circ}$, whereas AQSE [blue (dark-gray) curve] does not follow the true value.

Due to the intrinsic stochastic nature of quantum mechanics, the trajectory differs every time, even for photons of the same state, so statistical analysis is necessary to evaluate the performance of the scheme. Figure 3(b) shows the simulation results averaged over 500 trajectories of SAQSE compared to AQSE. The horizontal axis indicates the photon number (n). The vertical axis shows the average of the estimated angle. These results clearly show that the estimated angle by SAQSE [orange (gray) curve] follows the true value (black dashed curve) over time whereas AQSE [blue (dark-gray)

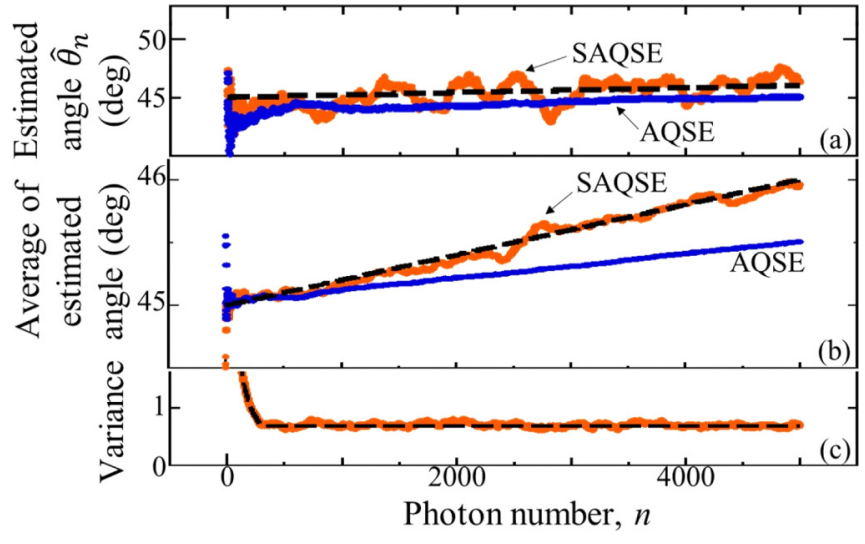

FIG. 3. Simulation results for AQSE and SAQSE. (a) Single trajectory of the simulation result of AQSE and SAQSE $(S=300)$. The black dashed line is the true value, the orange (gray) curve is the estimated values with SAQSE, and the blue (dark-gray) curve is the estimated values with AQSE. (b) Simulation results averaged over 500 trajectories of SAQSE $(S=300)$ compared to AQSE. The black dashed line is the true value, the orange (gray) curve is the estimated values with SAQSE, and the blue (dark-gray) curve is the estimated values with AQSE. (c) Variance when estimating 500 times with SAQSE $(S=300)$. The black dashed line is the CramérRao bound for a measurement with $n$ photons when $n<300$ and for a measurement with 300 photons when $n \geqslant 300$. The orange (gray) curve is the variance of each photon number when SAQSE is repeated 500 times.

curve] does not. Finally, the variance when estimating 500 times with SAQSE is shown in Fig. 3(c), showing that the variance of the SAQSE simulation results [orange (gray) curve] coincides with the optimal theoretical variance (black dashed line) called the Cramér-Rao bound for a measurement with 300 photons when $n \geqslant 300$.

Next, we numerically simulate SAQSE with true values changing with different constant rates in order to clarify the effect of the rate of change on the estimation. Figures 4(a)-4(c) show the simulation results averaged over 500 trajectories of SAQSE $(S=200)$ compared to AQSE. The horizontal axis indicates the photon number $(n)$. The vertical axis shows the average of the estimated angle. The amount of variation of the true value is monotonic and (a) $1^{\circ}$ for 200 input photons, (b) $2^{\circ}$ for 200 input photons, and (c) $4^{\circ}$ for 200 input photons. These results show that the angle estimated by SAQSE [orange (gray) curve] follows the true value (black dashed curve), but with a constant delay and the delay increases with the rate of change. The reason for the delay comes from our estimation procedure in which the recent $S \log$-likelihood functions are simply added without weight. As a result of the procedure, the estimated value coincides with an average of the recent $S$ true values. Thus, when the true value changes with a constant rate, it is expected that the estimated value will coincide with the past true value ( $S / 2$ photon number before). The yellow (light-gray) curves in Figs. 4(a)-4(c) denote the estimated values shifted by the $S / 2$ photon number. The yellow curves agree well with the true values. The variances when estimating 500 times with SAQSE for Figs. 4(a)-4(c) are shown in Figs. 4(d)-4(f), respectively. 

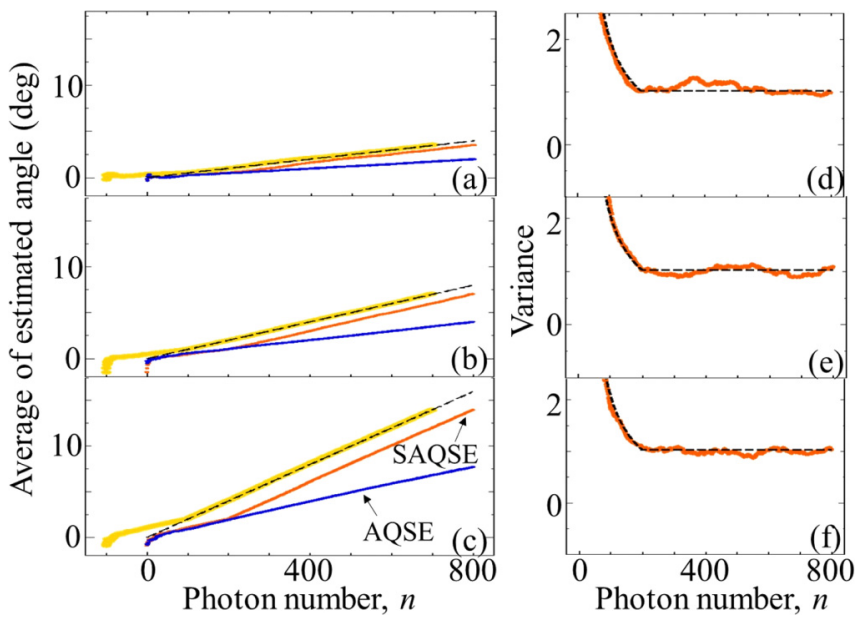

FIG. 4. Simulation results for SAQSE with the different rates of changes. (a)-(c) Average of estimated angle versus photon number. The black dashed line is the true value, the orange (gray) curve is the estimated values with SAQSE ( $S=200$ ), the blue (dark-gray) curve is the estimated values with AQSE, and the yellow (light-gray) curve is the estimated values shifted by $S / 2$. The amount of variation of the true value is (a) $1^{\circ}$ for 200 input photons, (b) $2^{\circ}$ for 200 input photons, and (c) $4^{\circ}$ for 200 input photons. (d),(e) Variance when estimating 500 times with SAQSE $(S=200)$. The black dashed line is the Cramér-Rao bound for a measurement with $n$ photons when $n<200$ and for a measurement with 200 photons when $n \geqslant 200$.

The variances of the SAQSE simulation results [orange (gray) curve] coincide with the optimal theoretical variance (black dashed line) called the Cramér-Rao bound for a measurement with 200 photons when $n \geqslant 200$.

\section{B. Simulation for sinusoidal changes of the true value}

In this section, we perform the numerical simulation of SAQSE for sinusoidally changing rates in order to clarify the effect of the changing rate on the estimation. Figure 5
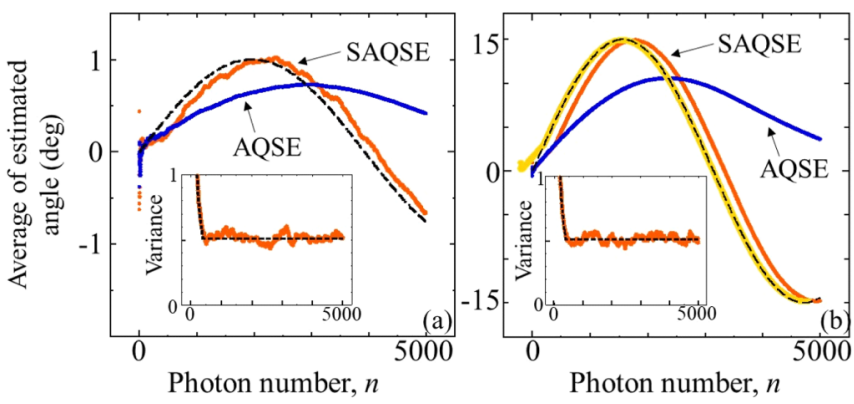

FIG. 5. Simulation results for SAQSE $(S=400)$ with the sine function. (a),(b) Average of estimated angle versus photon number. The black dashed curve is the true value, the orange (gray) curve is the estimated values with SAQSE, the blue (dark-gray) curve is the estimated values with AQSE, and the yellow (light-gray) curve is the estimated values shifted by $S / 2$. The insets show the variances when estimating 500 times with SAQSE. The black dashed line is the Cramér-Rao bound for a measurement with $n$ photons when $n<400$ and for a measurement with 400 photons when $n \geqslant 400$.

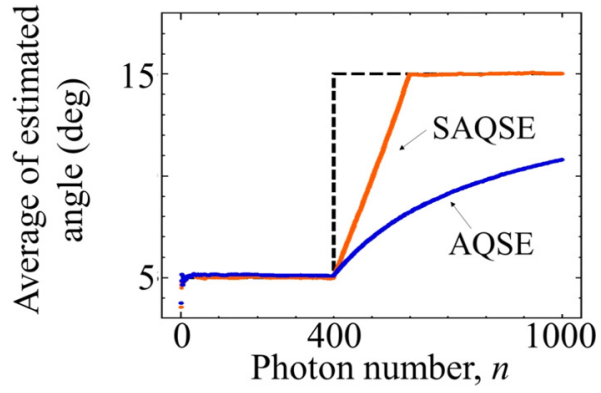

FIG. 6. Simulation results of SAQSE for a sudden change of the true value. The black dashed line is the true value, the orange (gray) curve is the estimated values with SAQSE $(S=200)$, and the blue (dark-gray) curve is the estimated values with AQSE.

shows the simulation results averaged over 500 trajectories of SAQSE compared to AQSE. The maximum amount of variation of the true value is (a) $0.32^{\circ}$ for 400 input photons $\left[\theta^{t}=\sin (0.144 n / \pi)[\mathrm{deg}]\right]$, (b) $5.8^{\circ}$ for 400 input photons $\left[\theta^{t}=15 \sin (0.184 n / \pi)[\mathrm{deg}]\right]$. The horizontal axis indicates the photon number. The vertical axis shows the average of the estimated angle. These results show that the angle estimated by SAQSE [orange (gray) curve] follows the true value (black dashed curve) over time whereas AQSE [blue (dark-gray) curve] does not. The estimated values have a delay of the $S / 2$ photon number from the true values for the same reason as discussed in the previous section. The yellow (light-gray) curve in Fig. 5(b) shows the estimated values [orange (gray) curve] shifted by the $S / 2$ photon number. The yellow curve agrees well with the true values (black dashed curve). The variances when estimating 500 times with SAQSE are shown in the insets in Figs. 5(a) and 5(b). The variances of the SAQSE simulation results [orange (gray) curve] coincides with the optimal theoretical variance (black dashed line) called the Cramér-Rao bound for a measurement with 400 photons when $n \geqslant 400$.

\section{Simulation for a sudden change of the true value}

Here, we numerically simulate SAQSE with the true value with a sudden change from a constant value to another constant value. Figure 6 shows the simulation results averaged over 500 trajectories of SAQSE compared to AQSE. True value is $5^{\circ}$ when $n<400$, and $15^{\circ}$ when $n \geqslant 400$. The horizontal axis indicates the photon number. The vertical axis shows the average of the estimated angle. The simulation result shows that the estimated value does not coincide with the true value shifted by $S / 2$ in this case. It is because, in this case, the averages of recent $S$ true values are not equivalent to the shifted true values.

\section{EXPERIMENTAL DEMONSTRATION}

We experimentally demonstrate SAQSE using the experimental setup in Fig. 2. In order to focus on the demonstration of the efficiency and the consistency of SAQSE, which are the most important features to be verified from the theoretical viewpoint, we only performed for a constant rate. We estimate the rotation angle $\theta^{t}$ (true value) of HWP2. The polarization 


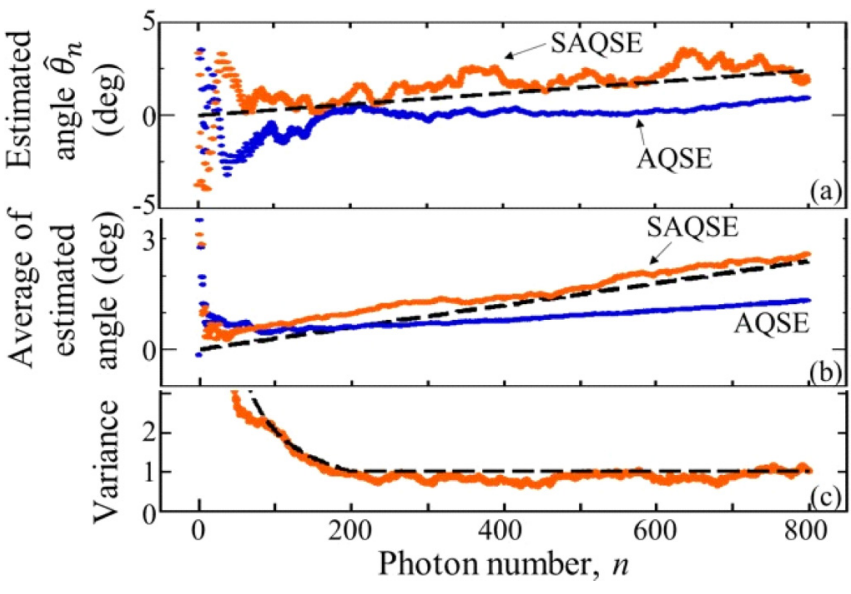

FIG. 7. Experimental results of AQSE and SAQSE $(S=200)$. (a) Single experimental trajectory of AQSE and SAQSE $(S=200)$. The black dashed line is the true value, the orange (gray) curve is the estimated values with SAQSE, and the blue (dark-gray) curve is the estimated values with AQSE. (b) Experimental results averaged over 100 trajectories of SAQSE $(S=200)$ compared to AQSE. (c) Variance when estimating 100 times with SAQSE $(S=200)$. The black dashed line is the Cramér-Rao bound for a measurement with $n$ photons when $n<200$ and for a measurement with 200 photons when $n \geqslant 200$. The orange (gray) curve is the variance of each photon number for SAQSE repeated 100 times.

of a photon is linearly changed. The amount of maximum variation in the angle is $0.6^{\circ}$ for 200 input photons. Figure 7(a) shows a single trajectory of the experimental result of SAQSE. The horizontal axis indicates the photon number $(n)$. The vertical axis shows the estimated angle of linear polarization $\hat{\theta}_{n}$. The experimental result shows that the angle estimated by SAQSE [orange (gray) curve] follows the true value (black dashed line) whereas AQSE [blue (dark-gray) curve] does not. Figure 7(b) shows the experimental results averaged over 100 trajectories of SAQSE compared to AQSE. The horizontal axis indicates the photon number $(n)$ and the vertical axis shows the average of the estimated angle. Similar to the numerical simulation, we randomly shift the measurement basis once every 30 times to suppress unexpected sudden changes in the estimated values and discard the unexpected sudden changes for the cases for which $\left|\hat{\theta}_{n}-\theta_{\text {true }}\right|>20^{\circ}$. The result is highly consistent with the simulation result and clearly shows that the estimated angle by SAQSE [orange (gray) curve] follows the true value (black dashed line) over time whereas AQSE [blue (dark-gray) curve] does not.

The variance when estimating 100 times with SAQSE is shown in Fig. 7(c), confirming that the variance of the SAQSE experimental results [orange (gray) curve] coincides with the optimal theoretical variance (black dashed line) called the Cramér-Rao bound for a measurement with 200 photons when $n \geqslant 200$.

Finally, we verify the consistency and efficiency of these experimental results. First we consider the estimation with SAQSE for four input states, $0.003 n, 20+0.003 n, 40+$ $0.003 n$, and $60+0.003 n$ [deg]. The amount of variation in each angle is $0.6^{\circ}$ for 200 photons input. This variation value is the same as for Fig. 7(a). Figure 8 shows experimental

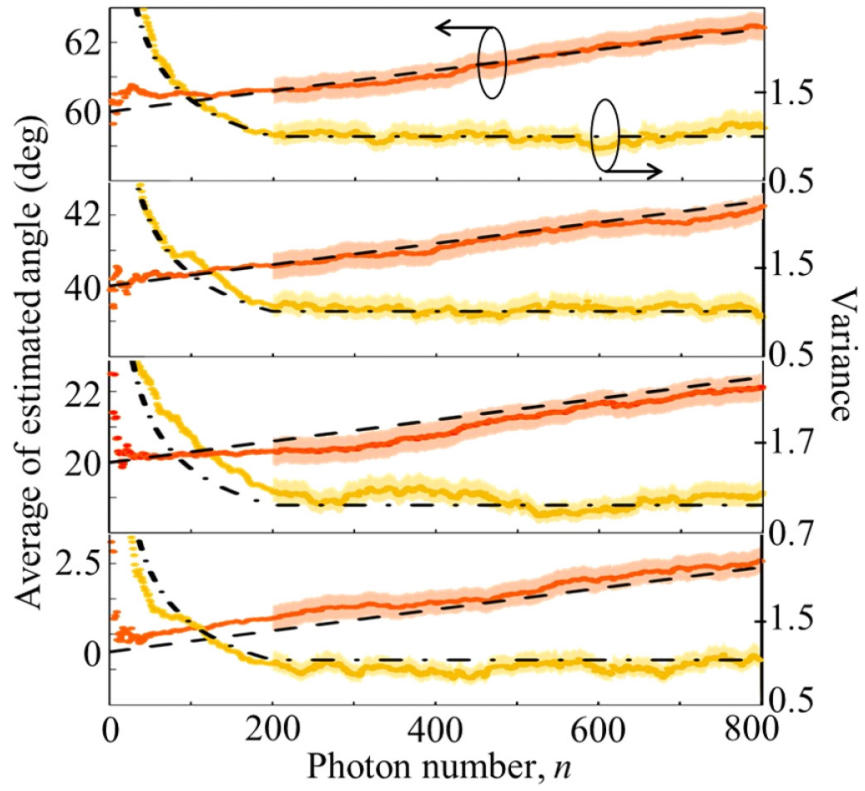

FIG. 8. Average of estimated values (left vertical axis) and variance (right vertical axis) versus photon number. Estimation results with SAQSE $(S=200)$ for four input states, $0.003 n, 20+0.003 n$, $40+0.003 n$, and $60+0.003 n$ [deg], are repeated 100 times and averaged over the 100 repetitions. The black dashed line is the input state, the orange (gray) curve is the estimated value of SAQSE $(S=200)$, and the orange shaded region indicates the area of the $90 \%$ confidence interval in the estimation results. The dash-dotted black line is the Cramér-Rao bound for a measurement with $n$ photons when $n<200$ and for a measurement with 200 photons when $n \geqslant 200$, the yellow (light-gray) dots are the variance of the estimated values, and the yellow shaded region indicates the area of the $90 \%$ confidence interval in the variance of the estimation results.

results with SAQSE $(S=200)$ over 100 repetitions. Note that the precision for preparing $\theta^{t}$ (true value) is about $\pm 0.2^{\circ}$, and the $90 \%$ confidence region (colored in orange) in Fig. 8 illustrates this precision. For the four cases, the $90 \%$ confidence region (colored in orange and yellow) overlaps well with the true value of the angle over time. These results confirm the consistency and the efficiency of the estimation in the proposed SAQSE method.

\section{CONCLUSION}

We have proposed an adaptive quantum state estimation scheme for nonstationary quantum states (SAQSE) by adopting the likelihood function for a fixed number $(S)$ of the most recent detection results. In the numerical simulation of SAQSE, we have shown that the estimated angles using SAQSE followed the true values with linear change, sinusoidal change, and sudden change whereas AQSE does not follow the true value. When the estimated value of SAQSE is shifted by $S / 2$, it well coincides with the true value of the slightly past state ( $S / 2$ photon number before) except for the case of the sudden change. In the experiments, we have shown that the estimated angles using SAQSE followed the true values over time well (consistency) while the former adaptive 
scheme (AQSE) was not consistent for various angles. We have also confirmed that the variances reached the CramérRao bound for the given number $S$ used for the likelihood function (efficiency). These results suggest that SAQSE can provide the most accurate estimation of unknown quantum states changing in time. Based on this, SAQSE may find useful applications not only in quantum information sciences, but other fields of science, such as astronomy and biology, where accurate estimation of quantum states is important.

\section{ACKNOWLEDGMENTS}

We would like to thank Dr. Koichi Yamagata of the University of Electro-Communications for the helpful discussions. We gratefully acknowledge financial support from MEXT/JSPS KAKENHI Grants No. JP26220712 and No. JP17H02861, JST-CREST Grant No. JPMJCR1674, MEXT Quantum Leap Flagship Program (MEXT Q-LEAP) Grant No. JPMXS0118067634, and a Grant-in-Aid for JSPS Fellows Grant No. JP18J22804.
[1] A. Soujaeff, S. Takeuchi, K. Sasaki, T. Hasegawa, and M. Matsui, J. Mod. Opt. 54, 467 (2007).

[2] A. Soujaeff, T. Nishioka, T. Hasegawa, S. Takeuchi, T. Tsurumaru, K. Sasaki, and M. Matsui, Opt. Express 15, 726 (2007).

[3] H. J. Kimble, Nature (London) 453, 1023 (2008).

[4] V. Scarani, H. Bechmann-Pasquinucci, N. J. Cerf, M. Dušek, N. Lütkenhaus, and M. Peev, Rev. Mod. Phys. 81, 1301 (2009).

[5] S. Takeuchi, Jpn. J. Appl. Phys. 53, 030101 (2014).

[6] M. Nielsen and I. Chuang, Quantum Computation and Quantum Information (Cambridge University Press, Cambridge, England, 2000).

[7] E. Knill, R. Laflamme, and G. J. Milburn, Nature (London) 409, 46 (2001).

[8] A. Aspuru-Guzik and P. Walther, Nat. Phys. 8, 285 (2012).

[9] S.-K. Liao et al., Nature (London) 549, 43 (2017).

[10] W. G. Cochran, J. Am. Stat. Assoc. 68, 771 (1973).

[11] H. Nagaoka, An asymptotically efficient estimator for a onedimensional parametric model of quantum statistical operators, Proceedings of the IEEE International Symposium on Information Theory (IEEE, 1988), p. 198.

[12] H. Nagaoka, On the parameter estimation problem for quantum statistical models, Proceedings of the 12th Symposium on Information Theory and Its Applications (SITA, 1989), pp. 577-582.
[13] H. Nagaoka, in Asymptotic Theory of Quantum Statistical Inference, edited by M. Hayashi (World Scientific, Singapore, 2005), pp. 125-132 (reprinted).

[14] A. Fujiwara, J. Phys. A: Math. Gen. 39, 12489 (2006).

[15] A. Fujiwara, J. Phys. A: Math. Theor. 44, 79501 (2011).

[16] R. Okamoto, M. Iefuji, S. Oyama, K. Yamagata, H. Imai, A. Fujiwara, and S. Takeuchi, Phys. Rev. Lett. 109, 130404 (2012).

[17] R. Okamoto, S. Oyama, K. Yamagata, A. Fujiwara, and S. Takeuchi, Phys. Rev. A 96, 022124 (2017).

[18] S. Nohara, R. Okamoto, K. Yamagata, A. Fujiwara, and S. Takeuchi, Jpn. J. Appl. Phys. 58, 072001 (2019).

[19] D. H. Mahler, L. A. Rozema, A. Darabi, C. Ferrie, R. BlumeKohout, and A. M. Steinberg, Phys. Rev. Lett. 111, 183601 (2013).

[20] K. S. Kravtsov, S. S. Straupe, I. V. Radchenko, N. M. T. Houlsby, F. Huszár, and S. P. Kulik, Phys. Rev. A 87, 062122 (2013).

[21] Z. Hou, H. Zhu, G.-Y. Xiang, C.-F. Li, and G.-C. Guo, npj Quantum Inf. 2, 16001 (2016).

[22] B. Qi, Z. Hou, Y. Wang, D. Dong, H.-S. Zhong, L. Li, G.-Y. Xiang, H. M. Wiseman, C.-F. Li, and G.-C. Guo, npj Quantum Inf. 3, 19 (2017).

[23] G. I. Struchalin, I. A. Pogorelov, S. S. Straupe, K. S. Kravtsov, I. V. Radchenko, and S. P. Kulik, Phys. Rev. A 93, 012103 (2016). 